\title{
Personalized medicine for cystic fibrosis: the next generation
}

\section{"If a genotype-directed cystic fibrosis transmembrane conductance regulator modulator is approved for use in cystic fibrosis, unprecedented opportunities will occur and new challenges may emerge."}

\section{KEYWORDS: ataluren $\approx$ CFTR $\approx$ cystic fibrosis $\approx$ genotype $\approx$ modulator $\approx$ personalized medicine $\approx$ potentiator $\approx$ therapy $\approx$ VX-770 $\approx$ VX-809}

The field of personalized medicine, based on genetic information, represents a tremendous but largely unfulfilled opportunity for a future generation of therapies [1]. While better clinical outcomes have been realized using predictive biomarkers in some forms of cancer [2], the influence of genetic information on the treatment of most other human diseases has been limited. However, in the case of the inherited genetic disease cystic fibrosis (CF), pre-emptive possibilities have recently been uncovered. In particular, an investigational drug known as VX-770, has demonstrated great promise in clinical trials in patients with a specific cystic fibrosis transmembrane conductance regulator $(C F T R)$ allele selected for clinical investigation based on data from in vitro studies [3,4]. This article highlights some of the opportunities and challenges associated with developing novel genotype-directed therapies for CF.

\section{The development of CFTR \\ genotype-directed therapies for CF}

Cystic fibrosis is a rare, life-shortening genetic disease primarily involving the exocrine pancreas, lungs and sweat glands. The original description was published more than 70 years ago, when little could be done to prevent mortality, the common outcome for infants and children afflicted at the time. Fortunately, patient survival has improved dramatically in recent years with the implementation of early diagnosis and aggressive care regimens directed at pancreatic and pulmonary symptoms [5].

Novel opportunities for clinical intervention were anticipated in 1989, when the CFTR gene was identified and several disease-causing mutations were found $[5,101]$. Determination of the CFTR genotype soon became a critical part of the CF diagnostic process, complementing the quantification of the CFTR protein's chloride channel function (e.g., by sweat chloride concentration) and the presence of typical symptoms [6]. Yet until now, the CFTR genotype has been of little use in the management of the disease, which has been focused on symptoms, not the underlying cause [5].

Among the hundreds of CF-causing CFTR mutations that have been identified, the F508del CFTR allele is the most common and is present in the great majority of patients in the USA and worldwide $[7,101]$. However, despite many individuals harboring this common allele, clinical phenotypes vary widely [5-7]. A number of different drugs are available to treat the panoply of CF-associated symptoms; however, there is no cure for this devastating disease and the median age of death in the USA remains $<30$ years of age [7].

The identification of CFTR more than 20 years ago led to a widespread hope that gene therapy would cure CF, but this promise is yet to be fulfilled. As efforts to find disease-modifying therapies have continued $[8,9]$, several factors have set the stage for the evolution of genotypedirected therapies. Well-validated methodologies have been available for years to determine CFTR genotypes. Consequently, in the US, nearly $90 \%$ of individuals with CF in the US Cystic Fibrosis Foundation (CFF) Patient Registry have had their genotype determined [7]. Valuable correlations have been made between some of the most common CF-causing CFTR genotypes and their clinical and cellular phenotypes. These data have provided important clues regarding how much CFTR activity may be needed to result in clinical benefit $[5,6,9]$.

Importantly, the CFF and its therapeutics arm, Cystic Fibrosis Foundation Therapeutics (CFFT), have propelled the field of therapeutic
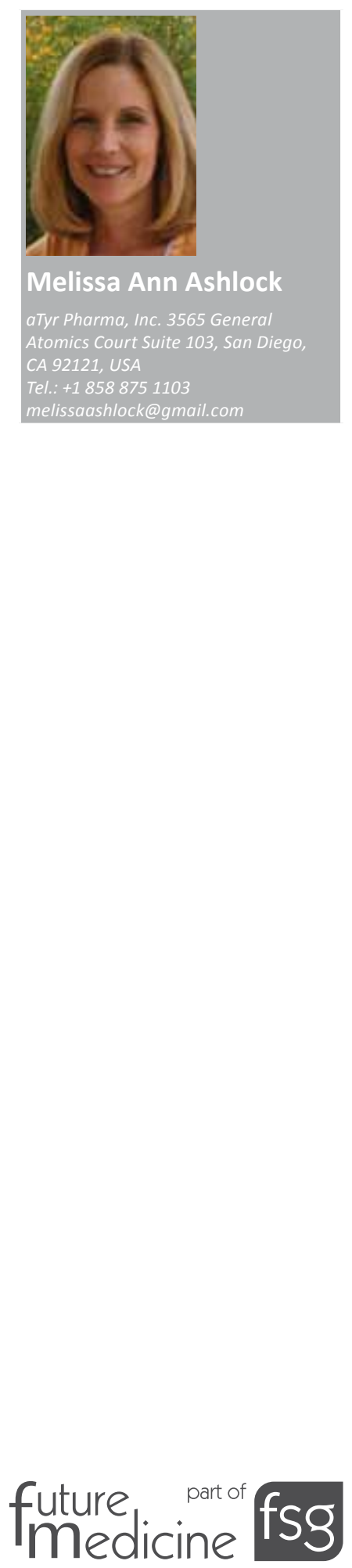
development forward by pioneering venture philanthropy to fund CFTR modulator programs within the pharmaceutical industry [9]. Among the companies studying genotypedirected therapies and receiving support from CFFT is PTC Therapeutics, Inc., whose lead compound, Ataluren is a small molecule that suppresses premature termination codons. Ataluren has been studied in Phase II clinical trials in CF subjects with CFTR nonsense mutations $[10,11]$, and is now undergoing Phase III investigation in CF patients [102]. Vertex Pharmaceuticals Inc. has received substantial support from CFFT (nearly \$75 million over the past 12 years) to pursue multiple avenues to improve CFTR dysfunction [9]. Clinical trials with Vertex's 'potentiator' of CFTR chloride channel gating (VX-770) have resulted in the most promising data among CFTR modulators investigated to date. In randomized, doubleblind, placebo-controlled Phase II and Phase III clinical trials, remarkably positive results were observed in both adults $[3,103]$ and children [104] with the G551D CFTR genotype, including an excellent safety profile and significant improvements in the primary Phase III clinical end point (lung function) and secondary end points such as respiratory exacerbation rate, bodyweight and sweat chloride concentration. The Phase III data have provided the first evidence of a sustained pharmacologic effect on CFTR from an investigational small molecule in $\mathrm{CF}$ and has generated a great deal of interest in the CF community and beyond [105].

\section{Preparing for the next generation of personalized medicine in CF - Using CFTR modulators in patients with qualifying genotypes}

If a genotype-directed CFTR modulator is approved for use in CF, unprecedented opportunities will occur and new challenges may emerge. Because the 'qualifying' CFTR genotype will be the major driver in the use of such a drug, a major question will be, how will clinicians also use clinical data when prescribing these therapies? This issue will be especially relevant for individuals with qualifying genotypes, but whose phenotypes (e.g., extremes of age or lung function) fall outside of those studied in Phase III clinical trials. Well delineated plans to make the drug available, perhaps on a 'compassionate use' basis and to investigate its safety and efficacy in phenotypes beyond those already studied in clinical trials, could help to quell concern over these issues.
Given that an effective CFTR modulator could transform patients' lives, the CF community must be prepared to address - and take advantage of - the consequences. Patients receiving effective CFTR modulators may feel better than ever before, leading them to try new activities. However, they may also attempt to reduce their existing treatment regimens, with the risk of generating untoward effects. Clinicians will need clinical data on the impact of changes in concomitant medications in the presence of CFTR modulators, to fully discuss with these patients whether it is appropriate to alter existing treatments regimens. In addition, an effective CFTR modulator may offer patients a new sense of hope for their future; however, having spent their entire lives battling this fatal disease, they may be poorly prepared to take advantage of their new potential. The various stakeholders in the therapeutic development for CF will need to anticipate these important life changes, and consider how to help.

\section{"The US Cystic Fibrosis Foundation and its therapeutics arm, Cystic Fibrosis Foundation Therapeutics (CFFT), have propelled the field of therapeutic development forward by pioneering venture philanthropy to fund CFTR modulator programs within the pharmaceutical industry."}

Clinical studies with CFTR modulators thus far have focused on well known clinical end points, especially those related to pulmonary disease and nutrition $[3,10,11]$. However, because CFTR is a dysfunction associated with many different organs and cellular abnormalities [9], other aspects of patients' clinical profiles could change when CFTR function is restored. For example, if a CF medication (e.g., inhaled antibiotic) targets a process (e.g., airway infection), which improves with restoration of CFTR function, the medication may no longer be needed or its dose may need adjustment. If CFTR function plays a role in the altered pharmacokinetics observed with certain drugs used in CF (e.g., aminoglycosides [12] and immunosuppressive agents [13]), dose adjustments of these drugs may be required to maintain their efficacy and/or prevent toxicity. If the presence of a comorbidity (e.g., diabetes) [5,9] is driven to by CFTR dysfunction, the need to treat that condition may change. However, if development of the comorbidity is influenced by a specific genetic modifier other than CFTR [14], 
the modifier gene itself - or its protein product - may need to be modulated to minimize the associated phenotype.

\section{Expanding the use of CFTR} modulators to patients with other CFTR genotypes

To date, CFTR modulators have been studied in only a small proportion of the $\mathrm{CF}$ population with a few of the most common CFTR mutations. In the past, to select the particular mutations to test, and to design clinical trials with different categories of CFTR modulators (e.g., potentiators and correctors), investigators have considered genotype-phenotype correlations between some relatively common mutations and their CFTR function in vivo (e.g., sweat chloride) and in vitro (e.g., cAMP-stimulated chloride channel function with and without the CFTR modulator) $[4,15]$. Recently, additional correlative data have been generated with less common mutations, including others beyond G551D CFTR that are associated with impaired CFTR channel gating $[16,17]$. Remarkably, these in vitro data from mammalian cell systems indicate that VX-770 can increase the CFTR function associated with these mutations to levels observable in the presence of G551D CFTR, suggesting that patients with these mutations could also benefit from VX-770.

However, the path to clinical trials of VX-770 in patients with other less common mutations is not yet clear. In the past, Vertex utilized a human airway epithelial model system that employed primary airway cells from lung transplant patients harboring the selected mutations to assess CFTR modulator efficacy in vitro and attempt to predict in vivo responses. In fact, this model system was rather predictive of the magnitude of the in vivo CFTR functional responses to VX-770 in patients with G551D CFTR [3,4,103], and in various other clinical trials of VX-770 and VX-809 (a corrector of CFTR protein folding and function [15]) in patients homozygous for F508del CFTR $[18,19,106]$. However, because it will be difficult to obtain primary airway cells from individuals with less common CFTR mutations (including other gating mutations), the model as it stands will likely be of limited utility. The fact that there is no validated alternative to the human airway cell model system will need to be addressed.

A major question will be how to advance a molecule like VX-770 into additional clinical trials with other CFTR genotypes, when pharmacodynamics assays are limited and the small number of possible participants makes it difficult to perform robust, well-powered Phase III clinical trials. As the CF community seeks solutions to these questions, the heightened interest by federal agencies to expedite drug development for small patient populations with rare genetic diseases could be especially important $[1,20]$.

\section{Basic \& clinical research opportunities}

Some key questions related to the pathogenesis of CF could also be addressed if CFTR modulators were available. These include whether organ failure in CF can be reversed, delayed or prevented, and whether lifespan can be extended. It will also be important to understand at what stage of CF a CFTR modulator needs to be given - and for how long - to alter the course of this chronic disease. If it were possible to study CFTR modulators in infants diagnosed by newborn screening, among other applications the potential of preventing the development of lung disease or eliminating the need for pancreatic enzymes could be assessed. Whenever possible, CF animal models should be used to complement these studies (to shed light on the role of CFTR in development) [9], and to realize the potential of modulators to have an even greater impact on outcomes, if administered before birth.

\section{"With genotype-directed therapies on the horizon in $C F$, the door is opening to the next generation of personalized medicine."}

A point for future discussion concerns whether extensive genotype information beyond CFTR should be obtained from patients. The purpose of obtaining such data could include complementing clinical information in patient registries to lead to a greater understanding of the genetic basis of differences in drug responsiveness and metabolism and a better informed selection of patients for clinical trials. While the contribution of known CF modifier genes or other genes on variation in response to CFTR modulators among patients $[3,10,11]$ is not yet known, the importance of considering an overlap between the responses and various genotypes may be increased by recent findings [21]. The study relates to $T L R 5$ c. $1174 \mathrm{C}>\mathrm{T}$, a mutant allele of TLR 5 which is found in approximately $6 \%$ of Canadian patients and codes for a premature stop codon. The data indicate that the allele is associated with altered inflammatory responses in stimulated lymphocytes, and suggests that this allele confers a better nutritional 
status among patients in the clinical setting. Additional studies are necessary to confirm the clinical association; however, because both inflammatory responses and nutritional status are assessed as outcomes in CFTR modulator trials, it could be important to consider whether potential modifiers such as TLR5 c. $1174 \mathrm{C}>\mathrm{T}$ are present in individuals who receive CFTR modulators, and whether the particular genotype influences drug responses. It could be worthwhile to configure new in vitro assays to evaluate the impact of this allele and other potential genetic modifiers, on the biologic activity of CFTR modulators with various modes of action.

In summary, with genotype-directed therapies on the horizon in $\mathrm{CF}$, the door is opening to the next generation of personalized medicine. In order to truly realize the potential of this opportunity, substantial and continual efforts will be needed. However, the potential rewards for patients and clinicians should be well worth the while, as successful implementation could dramatically improve the lives of CF patients and have a positive impact on a much broader class of diseases. As individuals see the benefits of these efforts, they may come to expect more tailored approaches for all of their medical care in the future.

\section{Acknowledgements}

The author thanks Allen Lapey, William Balch, Elizabeth Joseloff, Melanie Leitner, John McKew, Eric Olson and Hebe Quinton for their editorial comments and Carlo Castelani, Mitchell Drumm, Garry Cutting, Michael Knowles, Paul Negulescu, Stuart Peltz, Felix Ratjen and Richard Simon for helpful discussions.

\section{Financial \& competing interests disclosure}

In the past year, Melissa Ashlock has received consulting fees from Cystic Fibrosis Foundation Therapeutics, PTC Therapeutics, Inc. and Vertex Pharmaceuticals, Inc. She owns stock in Vertex. The author has no other relevant affiliations or financial involvement with any organization or entity with a financial interest in or financial conflict with the subject matter or materials discussed in the manuscript apart from those disclosed.

No writing assistance was utilized in the production of this manuscript.

\section{References}

1 Hamburg MA, Collins FS. The path to Personalized Medicine N. Engl. J. Med. 363(4), 301-304 (2010).

2 Ma BBY, Loong H. Personalized cancer therapy coming of age: clinical highlights in 2009 and future directions. Personalized Medicine 7(2), 121-124 (2010).

3 Accurso FJ, Rowe SM, Clancy JP et al. Effect of VX-770 in persons with cystic fibrosis and the G551D-CFTR mutation. N. Engl. J. Med. 363(21), 1991-2003 (2010).

4 Van Goor F, Hadida S, Grootenhuis PD et al. Rescue of CF airway epithelial cell function in vitro by a CFTR potentiator, VX-770. Proc. Natl Acad. Sci. USA 106(44), 18825-18830(2009).

5 Cystic Fibrosis in the 21st Century. Progress in Respiratory Research (Volume 34). Bush A, Alton EW, Davies JC, Griesenbach U, Jaffe A (Eds). Karger Publishers, Basel, Switzerland (2006).

6 Moskowitz SM, Chmiel JF, Sternen DL et al. Clinical practice and genetic counseling for cystic fibrosis and CFTR-related disorders. Genet. Med. 10(12), 851-868 (2008).

7 Cystic Fibrosis Foundation Patient Registry. Annual Data Report. Cystic Fibrosis Foundation, MD, USA (2010).

8 Ashlock MA, Beall RJ, Hamblett NM et al. A pipeline of therapies for cystic fibrosis. Semin. Respir. Crit. Care Med. 30(5), 611-626 (2009).
9 Ashlock MA, Olson ER. Therapeutics development for cystic fibrosis - a successful model for a multi-system genetic disease. Annu. Rev. Med. 62, 107-125 (2011).

10 Sermet-Gaudelus I, de Boeck K, Casimir GJ et al. Ataluren (PTC124) induces CFTR protein expression and activity in children with nonsense mutation cystic fibrosis. Am. J. Respir. Crit. Care Med. 182(10), 1262-1272 (2010).

11 Kerem E, Hirawat S, Armoni S et al. Effectiveness of PTC124 treatment of cystic fibrosis caused by nonsense mutations: a prospective Phase II trial. Lancet 372(9640), 719-727 (2008).

12 Tan KH, Mulheran M, Knox AJ, Smyth AR. Aminoglycoside prescribing and surveillance in cystic fibrosis. Am. J. Respir. Crit. Care Med. 167(6), 819-823 (2003).

13 del Tacca M. Prospects for personalized immunosuppression: pharmacologic tools - a review. Transplant Proc. 36(3), 687-689 (2004).

14 Cutting GR. Modifier genes in Mendelian disorders: the example of cystic fibrosis. Ann. NY Acad. Sci. 1214, 57-69 (2010).

15 van Goor F, Hadida S, Grootenhuis PD et al. Rescue of the protein folding defect in cystic fibrosis in vitro by the investigational small molecule, VX-809. J. Cyst. Fibros. (Suppl. 1) (2010).
16 van Goor F, Yu H, Burton B et al. Investigational compound V-770 potentiated multiple CFTR channel gating mutants in vitro. J. Cyst. Fibros. 10 (Suppl. 1), S17 (2011) (Abstract 62).

17 Sosnay PR, Castellani C, Corey M et al. Evaluation of the disease liability of CFTR variants. Methods Mol. Biol. 742, 355-372 (2011).

18 Clancy JP, Rowe S, Accurso FJ et al. Results of a Phase IIa study of VX-809, an investigational CFTR corrector compound, in subjects with cystic fibrosis homozygous for the F508del-CFTR mutation. Thorax (2011) (Epub ahead of print).

19 Flume PA, Borowitz DS, Liou TG et al. VX-770 in subjects with cystic fibrosis who are homozygous for the F508del-CFTR mutation. J. Cyst. Fibros. 10(Suppl. 1), S17 (2011) (Abstract 61).

20 Coté TR, Xu K, Pariser AR et al. Accelerating orphan drug development. Nat. Rev. Drug Discov. 9(12), 901-902 (2010).

21 Blohmke CJ, Park J, Hirschfeld AF et al. TLR5 as an anti-inflammatory target and modifier gene in cystic fibrosis. J. Immunol. 185(12), 7731-7738 (2010).

\section{Websites}

101 Cystic Fibrosis Centre, Hospital for Sick Children. Cystic fibrosis mutation database www.genet.sickkids.on.ca/app (Accessed 23 June 2011) 
102 PTC Press release: PTC Therapeutics Initiates Registration-Directed Phase 3 Trial of Ataluren in Cystic Fibrosis www.prnewswire.com/news-releases/ ptc-therapeutics-initiates-registrationdirected-phase-3-trial-of-ataluren-in-cysticfibrosis-62128492.html

(Accessed 23 June 2011)

103 Vertex press release: Phase III STRIVE study of VX-770 showed durable improvements in lung function (FEV1) and other measures of disease among people with a specific type of cystic fibrosis

http://investors.vrtx.com/releasedetail.

$\mathrm{cfm}$ ? releaseid $=583934$

(Accessed 23 June 2011)
104 Vertex press release: Phase 3 study of VX-770 in children ages 6 to 11 with a specific type of cystic fibrosis showed profound improvements in lung function (FEV1) and other measures of disease through 24 weeks http://investors.vrtx.com/releasedetail. cfm? releaseid $=560382$

(Accessed 29 March 2011)

105 Bloomberg Businessweek Vertex's treatmen for cystic fibrosis: its VX-770 is the first pill designed to repair an inherited flaw based on the discovery of the gene mutation that causes the disease

www.businessweek.com/magazine/ content/11_13/b4221061268683.htm (Accessed 17 March 2011)
106 Vertex press release: Interim phase 2 data showed a combination of VX-770 and VX-809 improved function of the defective protein that causes cystic fibrosis in people with the most common form of the disease http://investors.vrtx.com/releasedetail. cfm? releaseid $=583683$

(Accessed 9 June 2011) 